(Aus den niederösterreichischen Landesanstalten für Nerven- und Geisteskranke „Am Steinhof" in Wien [Direktor: Regierungsrat Dr. H. Schlöss].)

\title{
Psychologische Betrachtungen über Intellekt und Willen und deren Bedeutung in normalen und pathologischen Bewußtseinszuständen.
}

\author{
Von \\ Primarius Dr. K. Edler v. Wieg-Wickenthal, \\ Chefarzt der Frauenheilanstalt „Am Steinhof“, Gerichtspsychiater in Wien.
}

(Eingegangen am 1. Oktober 1914.)

Die psychiatrische Wissenschaft hat sich nicht nur zur Aufgabe gestellt, psychische Krankheitsbilder zu beschreiben und zu studieren, um dadurch beim geistig erkrankten Menschen Anhaltspunkte für ein ärztliches Wirken zu finden, sondern sie ist eben in Verfolgung dieses letzten Zieles auch bemüßigt, die krankhaften Vorgänge im Fühlen, Wollen und Denken des Menschen kritisch zu beleuchten, um erst dadurch Verständnis für die gestörten psychischen Funktionen anzubahnen und $\mathrm{zu}$ vermitteln. Ihr Wirkungsgebiet gehört also ebenso der praktischen Medizin wie der Psychologie an, sofern diese als Zweig der allgemeinen Biologie aufgefaßt wird.

Meines Erachtens - und vielleicht stimmen viele Fachkollegen mit mir darin überein — ist ein tieferes Verständnis der Psychosen nur durch ein gründliches psychologisches Vorstudium möglich, und darauf hingeleitet werden wir ja gerade durch diejenigen Psychologen und Philosophen (Wundt, Fechner), die den Begriff des psychophysischen Parallelismus in die Psychologie eingeführt und damit wieder der Reihe des psychischen Geschehens in ihrer Selbständigkeit zu ihrem Rechte verholfen haben. Die Aufstellung der Tatsache des psychophysischen Parallelismus wurzelt bekanntlich in der These, daß jedem elementaren Vorgange (Zustandsänderung) auf psychischem Gebiete ein solcher auf somatischem entspricbt, wobei aber immer die somatischen und psychischen Reihen scharf geschieden bleiben und, bis jetzt wenigstens, keine Brücke vom Somatischen in das Gebiet des Geistigen fübren kann. Die somatische, materielle Reihe (somatischer Parallelvorgang) ist naturwissenschaftlich deskriptiver Erforschung zugänglich, die psychische Reihe ist aber nur von dem Standpunkte innerer Erfahrung und inneren Erlebens aus, also durch psychologische Forschung zu ergründen. 
Die folgenden Zeilen sollen nun einen kleinen Beitrag zu allgemeinen psychologischen Fragen insbesondere, sofern sie uns in ihrer Anwendung auf die Psychopathologie interessieren, liefern.

In der heutigen Psychologie stehen sich, wie bekannt, zwei Richtungen scharf gegenüber: einerseits die Vertreter der Assoziationspsychologie, die unser ganzes psychisches Leben aus Empfindung und Vorstellung erklären wollen, den Gefühlston als eine der Empfindung immanente Eigenschaft ansehen und von einer eigentlichen Willenshandlung, überhaupt von einer Willensfunktion nichts wissen wollen, anderseits $W$ undt und seine Schule, die zur Erklärung der psychischen Funktionen den Begriff der Apperzeption, einer Willenshandlung, die mit dem subjektiven Gefühle der Aufmerksamkeit und muskulären Spannungsempfindungen einhergeht, einführte und in dieser das dominierende Prinzip, das Aktivitätselement des bewußten Psychismus erkannte.

Im folgenden will ich zu zeigen versuchen, daß Empfindung und Vorstellung, die eigentlichen Substrate für unseren Intellekt, in unserem Seelenleben, insbesondere im gestörten, eine ganz untergeordnete Rolle spielen und daß die weitaus wichtigsten und viel häufigeren Störungen aus krankhaften Willensfunktionen abzuleiten sind, daß also das voluntaristische Prinzip in der Psychologie und Psychopathologie von eminentester Bedeutung ist. Der Begriff Wille soll hierbei durchaus nicht in metaphysischem Sinne gebraucht werden, sondern, indem wir uns strenge an den empirischen psychologischen Willensbegriff halten, beabsichtigen wir zu zeigen, daß eben bei der Mehrzahl der geistigen Störungen dieses uns aus innerster Erfahrung bekannte Aktivitätsgefühl und Tätigkeitsmoment, das jedes bewußte psychische Geschehen begleitet und den subjektiven Ausdruck für die in uns wirkende Willensfunktion darstellt, Schaden leidet, wodurch tiefe Störungen in der Bewußtseinstätigkeit eintreten, die sich klinisch als das Krankheitsbild verschiedener Psychosen kundgeben.

Die Grundlage für unseren Intellekt liefern uns bekanntlich einzig und allein unsere Sinnesempfindungen und die daraus sich ableitenden Vorstellungen. In ihnen spiegelt sich die objektive Außenwelt gewissermaßen wider, wodurch wir erst Kenntnis von derselben, als der „Welt der Erscheinungen" erlangen. Jede Einwirkung seitens der Außenwelt affiziert aber gleichzeitig auch unser eigenes Ich, wird also als Einwirkung auf das Subjekt empfunden und ruft so eine subjektiv fühlbare Reaktion, unabhängig von den der äußeren Objektivität entsprechenden Bestandteilen der Sinneswahrnehmungen und Vorstellungen, hervor. Der innerste Kern unseres Ich-Komplexes nimmt gewissermaßen für sich ganz subjektiv Stellung gegen die durch die Sinneswerkzeuge angezeigte Zustandsänderung im Sinne innerer Abwehr oder Zustimmung, 
welche Stellungnahme des Ichs von uns als Lust oder Unlust empfunden wird.

Diese Seite unseres Ich-Komplexes, die eben durch die Data des Intellekts (Empfindungen, Vorstellungen) unmittelbar und primär affiziert wird, stellt eigentlich das Innerste unseres Wesens dar. Es soll mit dieser Behauptung nichts anderes als die jedem einzelnen von der Natur mitgegebene innere Anlage, in bestimmter individueller Art auf Außenwirkungen zu reagieren, gemeint sein, die erfahrungsgemäß jedem Individuum erst in bezug auf sein ganzes künftiges psychisches Verhalten den Stempel des Persönlichen, geistig Individuellen aufdrückt, wie die äußeren Formen des Körpers die jedem einzelnen eigene Körperkonfiguration ausmachen. Daß dieser integrierende Bestandteil unseres Geisteslebens a priori nichts mit Verstand, Urteil und Vernunft, kurz nichts mit den intellektuellen Funktionen zu tun hat, könnte jeder aus eigenster innerer Erfahrung bei einiger Fähigkeit zur Selbstbeobachtung wissen. Der Fähigkeit zur Perzeptivität, d. h. zur Aufnahme von Sinnesempfindungen, die dann zu Vorstellungen und Vorstellungskomplexen und schließlich zu unserem gesamten intellektuellen Inventar führen, und der oben erwähnten Fähigkeit zur Rezeptivität, d. h. der Fähigkeit, die Einwirkungen der Außenwelt innerlich mitzuerleben, also durch dieselben im Sinne von Lust oder Unlust affiziert zu werden, liegen wohl nicht verschiedene psychische Vermögen, verschiedene Qualitäten unserer Psyche zugrunde, sondern sie sind, wie ich glaube, der einheitliche Ausdruck des der Entladung psychischer Energie fähigen lebenden Hirnprotoplasmas. Die Summe der aufgespeicherten psychischen Energie dürfte vom biologischen Standpunkte aus wieder in nichts anderem als in der dem lebenden Hirngewebe innewohnenden Vitalität oder dem Biotonus desselben zusammenzufassen sein. Man könnte sich die ganzen hier angedeuteten Verhältnisse insofern bildlich recht verständlich machen, als man gewissermaßen eine Dichotomie des psychischen Energiestromes, der der Sinnesempfindung zugrunde liegt, bei seinem Eintritt in das psychische Organ annimmt und sich vorstellt, daß die primäre Energiewelle sich in zwei Erregungswellen teilt, wovon die eine in das rein intellektuelle Gebiet abläuft, die andere zum Erreger für das Subjekt im Sinne von Gefühlsbetonung und Affektivität wird. Mit jeder Perzeption ist also nach dem Gesagten auch das affektive Moment infolge Reaktion des Subjektes auf die erstere unmittelbar verbunden, wiewohl natürlich nicht die Gefühlsbetonung der Vorstellung als immanenter Bestandteil beigegeben ist, sondern immer erst eine subjektive Reaktion des Individuums auf das innere Erlebnis hin bedeutet.

Der ganze Ablauf des bewußten psychischen Geschehens stellt dann eigentlich, um bildlich weiter zu sprechen, einen in sich geschlossenen 
Ring dar, an dessen "Anfang" die Sinnesempfindung und an dessen „Ende" der motorische Akt steht. Die Sinnesempfindungen führen nämlich, wie gesagt, zu Vorstellungskomplexen und zugleich zur affektiven Mitbeteiligung unseres Ich, also zur Affektivität (Gefühle, Affekte), die wieder zum treibenden Moment für unser Handeln wird und in dieser Entwicklungsphase eben Wille genannt wird, der uns subjektiv als Tätigkeitsgefühl, das mit muskulären Spannungsempfindungen verbunden ist, zum Bewußtsein kommt. In diesem Sinne soll auch in folgendem der psychologische Begriff „Wille“ nur als eine bestimmte Entwicklungsphase der allgemeinen Affektivität verstanden werden. Gefühl, Affekt, Wille sind dann eigentlich nur verschiedene Stationen auf derselben Strecke, nämlich auf der Strecke des affektiven psychischen Energiestromes.

Das psychische Geschehen in uns führt, sofern es sich in unserem Bewußtsein abspielt, entweder zu einem motorischen Endeffekt (willkürliche Handlung) oder läuft ohne sichtbare Wirkung nach außen ab (willkürliches Denken, Affckte). Beide Funktionen sind willkürliche Entäußerungen der einzelnen individuellen Psyche und spielen sich, wie gesagt, in dem Bewußtsein des einzelnen ab, in welchem sie auch als inneres Erleben bewertet werden. Außer dieser bewußten psychischen Tätigkeit (Psychisme superieur) zwingen uns bestimmte psychopathologische Zustände und auch Erfahrungen aus dem Gebiete der normalen Seelentätigkeit eine zweite Stätte psychischen Wirkens anzunehmen, die unter der Bewußtseinsschwelle liegt, also der unterbewußten Sphäre psychischer Tätigkeit (Automatisme mental) angehört, und von deren aktivem Schaffen wir nur die Endglieder der Assoziationsketten erkennen, sofern sie uns durch ihr Aufsteigen über die Bewußtseinsschwelle eben bewußt werden.

$\mathrm{DaB}$ wir ohne die Annahme eines sog. Unterbewußtseins (Gebiet der "latenten" Vorstellungen) nicht auskommen, wenn wir vorurteilsfrei und ungezwungen die Tatsachen, die uns die einfache Selbstbeobachtung einerseits und die Erfahrungen auf dem Gebiete der Psychopathologie andererseits lehren, erklären oder wenigstens halbwegs verstehen wollen, zeigen uns die psychopathologischen Erscheinungen bei der Hysterie, das alternierende Bewußtsein (second état, second life) bei Psychopathen, wo für das ,normale " erste Bewußtsein das zweite sog. "Nebenbewußtsein" immer den Charakter des Un- oder Unterbewußten haben muß, endlich auch die tagtägliche Erfahrung der momentanen blitzartigen, mit der Lösung eines Problems, eines Gedankenkomplexes, der uns in unserem wachen Bewußtsein intensiv beschäftigte, in innigstem Zusammenhange stehenden Einfälle und Gedanken, die ganz ohne unser bewußtes Zutun gleichsam wie von selbst aus un- 
serem Innern emporsteigen und uns durch ihr plötzliches, ganz unerwartetes Dasein (Bewußtwerden) freudig überraschen. Wir müssen also schon beim normalen Ablauf der psychischen Funktionen neben dem Hauptbewußtsein, dem Oberbewußtsein auch ein Unterbewußtsein oder Nebenbewußtsein unterscheiden, das in strengem Sinne des Wortes eigentlich kein Bewußtsein mehr ist, weil seine Funktionen für gewöhnlich wenigstens uns im ganzen und großen unbewußt bleiben und automatisch ablaufen. Noch klarer als im normalen psychischen Leben treten die unterbewußten seelischen Funktionen in gewissen psychischen Grenzfällen (pathologische Einfälle der Psychopathen) zutage.

Das Bewußtsein - wir meinen damit in folgendem immer das Hauptbewußtsein - wird unterhalten durch den beständig ablaufenden psychischen Energiestrom, der, um wieder bildlich zu sprechen, mit einem gewissen Gefälle und einer gewissen Breite (Intensität und Extensität des Bewußtseins) eine Assoziationsreihe nach der anderen erreicht und in Tätigkeit versetzt. Der Weg, den die jeweilige psychische Aktionswelle nimmt, wird ihr im normalen Geistesleben durch die innere und äußere Apperzeption gewiesen, als denjenigen Willensvorgang, der eigentlich die ganze Richtung des psychischen Energiestromes bestimmt; denn durch die Apperzeption werden erst gewisse Assoziationsreihen in Tätigkeit versetzt und so das willkürliche Denken und Wahrnehmen, das so charakteristisch mit dem Gefühl der Aufmerksamkeit und den somatischen Spannungsgefühlen verbunden ist, ermöglicht.

Das Bewußtseinsfeld (die „Funktionsbereitschaft" des Bewußtseins [Berze]) hat beim Geistesgesunden einen ganz bestimmten Intensitätsindex, welcher die im Aktionsstrom aufgespeicherte und in den Zeiteinheiten zur Wirkung gelangende Größe oder Menge psychischer Energie anzeigt. Dieser Intensitätsfaktor muß nun eine gewisse Größe haben, unter welche er nicht weiter sinken darf, soll das individuelle Bewußtsein jenen Grad von Klarheit (Intensität) besitzen, den wir für das normale Geistesleben zur unbedingten Voraussetzung machen müssen. Der Intensitätsindex des Bewußtseinsfeldes wäre nach Analogien bei anderen Energieformen am besten vielleicht mit dem Potentiale eines elektrischen Kraftfeldes zu vergleichen und würde wie dort direkt proportional sein dem Gefälle des psychischen Aktionsstromes, oder mit anderen Worten, dem Niveaunterschiede zwischen zwei Punkten des psychischen Energiefeldes. Das bewußte psychische Geschehen wird also um so energischer, kraftvoller und intensiver vor sich gehen, d. h. die Klarheit des Bewußtseins wird eine um so größere sein, je größer gewissermaßen das erwähnte Potentiale, der Kraftindex des psychischen Feldes ist. Den normalen Zustand, d. h. 
den zu normalem Denken und Handeln nötigen Grad von Höbe und Klarheit des individuellen Bewußtseins, glaube ich, hat Berze recht treffend in dem Ausdruck Bewußtseinstonus, Bioton us des Bew ußt seins zusammengefaßt, wobei der normalerweise nötige Bewußtseinsgrad eben als Tonus des Bewußtseins bezeichnet wird, wie wir ja schon seit langem mutatis mutandis von einem Nerven- und Muskeltonus zu sprechen gewohnt sind. Dieser Bewußtseinstonus des Geistesgesunden ist nun in seiner letzten Ursache auf die im lebenden Hirngewebe ständig vor sich gehenden chemischen Lebensprozesse zurückzuführen. Nur auf ein in normalem Tonus sich befindendes Bewußtsein kann die apperzeptive Tätigkeit, das eigentlich aktive Prinzip im Psychismus des einzelnen, einwirken. Die Apperzeption ist so eigentlich der Spiritus rector, das leitende Prinzip, das gleichzeitig die Bewußtseinshelle (Klarheit) beeinflußt und die einzelnen Assoziationsreihen, gewissermaßen die Instrumente, auf denen gedacht wird, durch Fortleitung der psychischen Reizwelle in Tätigkeit und Erregung versetzt und erhält.

Ich weiß sehr wohl, daß die Vertreter der Assoziationspsychologie behaupten, die Zielvorstellungen und Motivvorstellungen für das willkürliche Denken und Handeln unterscheiden sich nur durch bestimmte qualitative Attribute (Konstellation, Gefühlstöne) von den übrigen Vorstellungen des jeweiligen Bewußtseinsinhaltes und das Denken und Handeln des Menschen komme einfach nach bestimmten Assoziationsgesetzen zustande, indem die mit obigen Attributen eben ausgestatteten Vorstellungen eine dominierende, richtunggebende Führung im Bewußtsein übernehmen und so erst uns als Ziel- oder Motivvorstellung zum Bewußtsein gelangen. Wozu brauche man dann noch einen deus ex machina, eine neue qualitas occulta, die sog. Apperzeption, die ja nach den Deduktionen der Assoziationspsychologen anscheinend ganz unnötig wäre, da ja der bewußte Psychismus dann wirklich nichts anderes als ein maschinenmäßiges Getriebe wäre, wobei die prävalierenden Vorstellungen (Ziel-, Motivvorstellungen) einfach ganz ohne unser individuelles Zutun bestimmten Kraftzentren im maschinellen Betriebe $\mathrm{zu}$ vergleichen wären, von denen aus lebendige Kraft weiter geleitet würde über ebenfalls schon präformierte Bahnen (Assoziationsketten); das Individuum selbst wäre seinen inneren Erlebnissen gegenüber immer zur Rolle des passiven Zuschauers verdammt, während wir doch sehr wohl aus unserer allereigensten, inneren Erfahrung wissen, daß wir bei all diesen inneren Vorgängen gerade das Gefühl aktiver Tätigkeit haben und das Produkt dieser Tätigkeit eben als unser geistiges Eigentum ansprechen müssen. Der Grundirrtum, der zu solchen der inneren Erfahrung eigentlich direkt widersprechenden Behauptungen führen konnte, ist in dem Vorurteile von der Immanenz der 
Gefühlsbetonung der einzelnen Empfindungen und Vorstellungen zu suchen. Unter der Annahme einer den Vorstellungen immanenten Gefühlsbetonung erscheint allerdings der ganze Psychismus (Psychisme superieur) nichts weiter als ein in Gang gesetztes Räderwerk zu sein ohne individuelles Gepräge und ohne Aktivität des Subjektes. Die infolge ihres Gefühlstones prävalierenden Vorstellungen beherrschen ohne unser Zutun eigentlich allein den Ablauf und die Richtung des Assoziationsmechanismus. Nun beruht aber das ganze in der Phantasie errichtete Gebäude auf falschen Voraussetzungen. Die verschiedenen Vorstellungen und Empfindungen haben eben gerade, wie eingangs ausgeführt wurde, keinen ihnen immanenten Gefühlston, wie die Assoziationspsychologen behaupten, sondern der jeweilige Gefühlston, der eine Vorstellung begleitet, entspricht einer aktiven Reaktion der Psyche des Individuums auf die vom Intellekt ihm zugeführten Erlebnisse. Die intellektuellen Funktionen haben sich phylogenetisch aufsteigend bis zu dem Grade der Vollkommenheit des menschlichen Intellekts immer in der einen Zweckrichtung entwickelt, dem Individuum Nachrichten über die Außenwelt (Wahrnehmungen, Sinneseindrücke) zu geben, um es instand zu setzen, situationsgemäß und zweckmäßig sich zu verhalten und zu handeln. Der primitivere Intellekt, wie wir ihn auch bei den Tieren bereits ausgeprägt finden, ist auf das unmittelbare Erkennen und sinnliche Anschauen beschränkt, er ist imstande, Ursache und Folge, also allgemeine Kausalitätsbeziehungen zu beurteilen und herauszufinden, wenn sich ihm unmittelbar die entsprechenden Sinneseindrücke in einer sukzessiven Reihe von Erscheinungen darbieten. Der menschliche Intellekt ist dagegen imstande, nicht nur grob sinnlich, auf Anschauung beruhend zu denken, sondern er ist durch die Wortvorstellungen der Sprache so unendlich vervollkommnet, daß er auch mit ganz abstrakten Vorstellungen und allgemeinen Begriffen zu operieren versteht und so eigentlich erst im abstrakten begrifflichen Denken sein wahres und ihm angemessenes Arbeitsfeld findet. Schopenhauer schreibt ganz richtig die Fähigkeit zur unmittelbaren sinnlichen Erkenntnis, die Fähigkeit, Kausalitätsverhältnisse richtig zu erfassen, den Funktionen des Verstandes zu und scheidet streng davon die Aufgabe der Vernunft, die eben im abstrakten begrifflichen Denken, im Operieren mit Begriffen besteht. Der menschliche Intellekt hat also nur die Rolle eines Berichterstatters für das Subjekt übertragen bekommen, um dasjenige Quale unserer Psyche, das eben primär und noch außer dem Intellekt in uns wohnt, stetig über die Vorgänge der Außenwelt im laufenden zu erhalten. Das psychische Leben, der ganze Psychisme superieur des Individuums besteht eigentlich nur in einem beständigen Affiziertwerden des Subjektes durch die Data des Intellekts oder durch die in der Körperlich- 
keit vor sich gehenden vasomotorischen Gesamtveränderungen mit entsprechender Reaktion des Individuums im Sinne einer Willenshandlung oder eines Affektvorganges. Die Reaktionen des Subjektes auf die vom Intellekt gelieferten Vorstellungen und Wahrnehmungen werden nun erst den letzteren den sog. ,,immanenten" Gefühlston im Sinne der in dem einzelnen Individuum verkörperten inneren Anlage verleihen und damit dieselben in einem gegebenen Zeitmomente zu im Interesse des Subjekts gelegenen hochwertigen Ziel- oder Motivvorstellungen emporheben oder infolge negativer Gefühlsbetonung (Reaktion des Subjektes im ablehnenden Sinne) dieselben alsbald aus dem Bewußtseinsinhalt ausschalten.

Aus unseren kurzen Auseinandersetzungen erkennen wir bereits, daß unser Wollen, eine eigentlich immer zur Aktivität drängende psychische Tätigkeit, die den inneren Kern jedes Seelenlebens ausmacht (Sc ho penhauer), einerseits aktiv in unserem Psychismus zur Geltung kommt und da als apperzeptive Tätigkeit oder kurzweg Apperzeption, die immer nur als Willensvorgang verstanden werden muß, die führende Rolle übernimmt und so zu dem das Denken, bewußte Wahrnehmen (Erkennen) und bewußte Handeln auslösenden und anregenden Kraftmomente wird, anderseits aber auch als bestimmte Entwicklungsphase unserer Affektivität in obigem Sinne aufgefaßt in der rezeptiven Fähigkeit unserer Psyche begründet ist, nämlich in der Fähigkeit, auf einstürmende Sinneseindrücke und Gedanken im positiven oder negativen Sinn zu reagieren, woraus erst die Gefühlsbetonung der Vorstellungen abgeleitet werden konnte. Aber nicht nur durch Veränderungen in der Außenwelt, wie sie uns durch den Intellekt zugeführt werden, wird unser innerstes Wesen affiziert und mithin zur Reaktion angeregt, sondern auch durch die in jedem Zeitmomente vor sich gehenden feinsten Veränderungen im Gebiete unserer Körperlichkeit fließen uns Reizquellen zu, die psychisch unbewußt uns beeinflussen und als Grundton unserer jeweiligen Stimmungslage uns wieder gewissermaßen retrospektiv bewußt werden. Unter diesen feinsten Veränderungen in unserem Körper verstehe ich die fortwährend wechselnden Lebensvorgänge in der lebenden Substanz der kleinsten Capillaren, die ständigen respiratorischen, sekretorischen und vasomotorischen Veränderungen, oder kürzer ausgedrückt, den ständig wechselnden Biotonus im gesamten Vasomotorium.

Aus dem ganzen Vorhergehenden können wir bezüglich Intellekt und Willen und deren gegenseitiges Verhältnis folgende Schlußfolgerungen ableiten: Der Wille in oben auseinandergesetztem Sinne ist einerseits als aktives Prinzip in unserem Psychismus tätig und leitet in diesem Sinne als Apperzeption unsere ganze Bewußtseinstätigkeit, anderseits ist er seiner Entwicklung nach ausschließlich von unserer Affektivität 
abhängig, stellt nichts anderes dar als die zum aktiv treibenden Moment gewordenen Affekte und ist in letzter Linie auf die beständig wechselnden Veränderungen in unserer Körperlichkeit und auf die äußeren Sinneseindrücke, auf welche unser Ich vermöge seiner Wesenheit mit einer Gefühlsreaktion im Sinne von Lust oder Unlust antworten muB, zurückzuführen. Diese jeweiligen Reaktionen des affizierten Ichs werden uns als Stimmungslage oder, in gesteigertem Maße, als Affekte bewußt.

Unter intellektuellen Prozessen möchte ich die bewußten Wahrnehmungen und das bewußte Vorstellungsleben verstanden wissen, wobei es sich beim Vorstellungsleben nicht nur um Vorstellen von Objekten der Außenwelt und abstrakte Begriffe, sondern auch um ein Vorstellen von Affekten handelt, so daß also bereits vorgestellte Affekte ebenso unseren Willen anregen wie Vorstellungen anderer Art. Der Wille in obigem psychologischen Sinne, der in der Affektivität begründet und aus ihren Grundlagen hervorgegangen ist, spielt demnach im ganzen Psychismus die erste Rolle, und mit vollem Rechte kann man auch in psychologischer Bedeutung von einem Primat des Willens, wie Scho pen hauer dies für den metaphysischen Willensbegriff bewiesen hat, sprechen. Der Intellekt (Vernunft und Verstandesfähigkeiten, Residuen des Wahrnehmungsvorganges $=$ Vorstellungen, Gedächtnis) ist für sich allein überhaupt nie tätig, sondern funktioniert nur im Bewußtsein im Vereine und unter Leitung der Apperzeption, nachdem ja die Funktionen des normalen Bewußtseins ein einheitliches Kontinuum darstellen, in dem die intellektuellen und Willensfähigkeiten gleichzeitig zur Geltung kommen. Die psychischen Fähigkeiten kommen immer nur im engen Zusammenhange untereinander, also jede Vorstellung assoziiert mit Gefühls- und Willensmomenten und umgekehrt, im Seelenleben zur Geltung.

Die Qualität des Denkens, die geistige Kapazität und Wertigkeit des einzelnen wird nach dem Vorhergehenden demnach hauptsächlich von zweierlei Momenten abhängen: erstens einmal von dem zur Verfügung stehenden Material zum Denken überhaupt, $d$. h. je größer das Material zum Denken ist, je allumfassender, tiefer die allgemeine Bildung ist, desto geistig höher stehender wird das betreffende Individuum sein; denn es ist klar, daß von zwei verschiedenen Menschen derjenige uns durch seine geleistete Denkarbeit mehr in Erstaunen setzen wird, bei dem sich auf Grund seiner Wahrnehmungen, Empfindungen und Vorstellungen, also auf Grund dessen, was ihm seine Bildung gegeben hat, weitverzweigtere, leicht erregbare Assoziationsketten und Reihen in seinem Assoziationsorgan vorgebildet finden, weil diese gewissermaßen das ,"materielle“ Substrat zu den Denkoperationen abgeben, obne welohe das eigentliche psychische Agens (Apperzeption) ebensowenig wirken kann, wie der genialste Feldherr niemals einen Schlachtenplan wird verwirklichen können, ohne eine gewisse Anzahl ihm zur Verfügung ste- 
hender Truppen; zweitens aber wird es bei der Beurteilung der Qualität des Denkens nicht nur auf die Zahl der „Bausteine“ zu den Denkoperationen ankommen, sondern auch hauptsächlich auf den Lenker und Leiter, auf die Art des wirksamen Prinzips, d. h. auf das Quale der jeweiligen apperzeptiven Funktion. Je größer die psychische Energie ist, mit der die Apperzeption verbunden ist, je größer der sogenannte Kraftindex des Bewußtseinsfeldes, demnach je größer der Bewußtseinstonus des einzelnen ist, desto qualitativ höherstehender werden wir die Denkoperationen des Betreffenden zu bewerten haben. Eine geniale geistige Veranlagung würden wir nach dem Gesagten dann vor uns haben, wenn wir einerseits einen weitverzweigten, die verschiedensten Assoziationszentren des Gehirns in Verbindung setzenden Assoziationsmechanismus vorfinden, anderseits die apperzeptive Fähigkeit und der Biotonus des Bewußtseins in dem betreffenden Psychismus den denkbar höchsten Grad und damit das individuelle Bewußtsein den größten Kraftindex, den größten Grad von Helle und Klarheit erreicht hätte.

Außer den im vorhergehenden kurz besprochenen Tatsachen des bewußten Seelenlebens, der durch die Apperzeption geleiteten Bewußtseinstätigkeit, welche die Franzosen mit dem Ausdruck Psychisme superieur zusammenfassen, müssen wir noch eine unterbewußte Geistestätigkeit bereits im normalen Geistesleben anerkennen, die bei den verschiedensten psychopathologischen Zuständen, wie wir später sehen werden, aber auch schon normalerweise eine Rolle spielen und der innersten Erfahrung und Erkenntnis zugänglich sind. Tagtäglich können wir bei einiger Fähigkeit zu vorurteilsfreier Selbstbeobachtung konstatieren, daß bei Abnahme der Funktionen unseres Oberbewußtseins (wachen Bewußtseins) der psychische Automatismus, das psychische Wirken unterhalb der Bewußtseinsschwelle in unserem Innern reger wird und einzelne psychische Gebilde (Gedanken, Gefühle) aus der Tiefe des Unbewußten in unser schon halb und halb gelähmtes Bewußtsein emporsteigen läßt. So erklären sich die oft in rascher Aufeinanderfolge und meist untereinander ganz unzusammenhängenden Gedankenreihen, die in unser Bewußtsein einstürmen, wenn wir einzuschlafen beginnen, uns also in dem bekannten Halbschlummer befinden, wobei unser im wachen Zustande funktionierendes Bewußtsein noch nicht durch die im Organismus angehäuften Ermüdungsstoffe so tief gelähmt ist, um nicht noch imstande zu sein, stärkere Sinneseindrücke zu perzipieren, eventuell auch zu apperzepieren, wobei aber die Energie, der Biotonus des Bewußtseins, doch bereits so erheblich abgenommen hat, daß es nicht mehr imstande ist, die aus der unbewußten Sphäre der Geistestätigkeit immer wieder auftauchenden Gedanken zurückzudämmen und ihnen den Eingang ins Bewußtseinsfeld zu verwehren. Dieselben Beobachtungen können wir bei den verschiedenartigsten sonstigen Geisteszuständen 
machen, soferne denselben gemeinsam eine Abnahme der wachen Bewußtseinstätigkeit infolge Ermüdung der lebenden Hirnsubstanz zugrunde liegt. So „,verwirren" sich uns die Gedanken nach langer, anstrengender, mit voller Apperzeptionskraft vor sich gehender geistiger Arbeit, eben weil die Apperzeption, ein Attribut der lebenden Hirnmasse, allmählich erlahmt und wie jede Funktion des lebenden Protoplasmas sich eben mit der Zeit erschöpft, wenn nicht Ruhepausen zum Ausgleich der verbrauchten Energie eingeschaltet werden. Wir sagen: „es verwirren sich unsere Gedanken", weil wir selbst das Gefühl haben, daß wir die Zügel für weitere geistige Operationen verloren haben, daß wir ohne unser aktives Zutun und unfreiwillig ein Spiel unserer Gedanken geworden sind.

Diese zuletzt erwähnten Tatsachen führen uns eigentlich schon unmittelbar zu den Erscheinungen, wie sie uns in psychopathologischen Zuständen entgegentreten. Nach Groß besteht die Hauptfunktion des normalen Bewußtseins in der Koordination und dem Zusammenfassen der sukzessiven und synchronen inneren Erlebnisse zu einem gemeinsamen Ganzen, dem Kontinuum des Bewußtseins, was wir als Einheit des Bewußtseins fühlen. Diese Höchstfunktion des Bewußtseins ist der Ausfluß der ungeschwächt vonstatten gehenden apperzeptiven Tätigkeit, die durch ihre das Bewußtseinsfeld überstrahlende Energie es zugleich unmöglich macht, daß Glieder psychischer „Nebenreihen“ (aus dem Unbewußten) störend in den Ablauf der Bewußtseinstätigkeit eingreifen. Bei Abnahme dieser Höchstfunktion infolge Hypotonie des Bewußtseins und sekundärem Nachlassen der Apperzeption (Berze) ist gewissermaßen die Sperre zwischen Ober- und Unterbewußtsein weggefallen, die Kräfteindices beider Bewußtseinssphären haben sich einander genähert und die psychischen Nebenreihen können trotz ihrer geringen Energie das Bewußtseinsfeld bei ihrem Auftauchen ins Bewußtsein doch beleuchten, d. h. uns bewußt werden, weil die Energie des Hauptbewußtseins, die früher alles aus dem Unterbewußten Emporsteigende überstrahlte, an Intensität eben wesentlich abgenommen hat.

Von derartigen schon im normalen Psychismus begründeten Erfahrungen ausgehend können wir nunmehr auch leichter einen Schlüssel und ein psychologisches Verständnis für die verschiedensten Erscheinungen der Psychopathologie gewinnen. Die leichtesten Grade einer Alteration des biochemischen Gleichgewichtes der äußerst empfindlichen Hirnsubstanz, wie es zum normalen Ablauf der Bewußtseinsphänomene eben nötig ist, wird gerade zuerst zu Störungen der erwähnten psychischen Höchstfunktion führen; denn diese stellt gewissermaßen das feinste Reagens dar, auf Grund dessen wir den normalen Psychismus beurteilen können. Als materiellen Parallelvorgang für diese hochwertige psychische Energieleistung müssen wir uns die äußerst subtilen und kompli- 
zierten chemischen Veränderungen in den Zellen der lebenden Hirnmasse vorstellen, so daß also der psychischen Energie auf somatischem Gebiete bestimmte Zustandsänderungen im Biochemismus der lebenden Hirnsubstanz entsprechen, womit aber keineswegs gesagt werden soll, daß mit diesen angenommenen Alterationen der materiellen Reihe zugleich auch erschöpfend und verständlich das Wesen der psychischen Energie erkannt werden kann. Ich glaube vielmehr, das innerste Wesen dieser böchsten Energieform, die uns die Natur in ihrem unermeßlichen Reiche der Erscheinungen in so vollendetem Maße in dem menschlichen Bewußtsein vor Augen führt, wird den engbegrenzten Erkenntnisformen unseres Intellekts nie zugänglich sein und die alte, aber immer neue Frage nach dem Quale dieser Kraft wird von unserem Intellekt ebenso ungelöst bleiben, wie die Frage nach dem innersten Kern der in ihren Erscheinungsformen sonst so gut gekannten anderen Naturkräfte.

Die supponierten feinsten biochemischen Veränderungen des Hirngewebes, die zu den erwähnten Störungen der höchsten Funktionen des Bewußtseins Anlaß geben sollen, können natürlich durch die verschiedensten exogenen Schädlichkeiten, also vor allem durch allgemeine Stoffwechselstörungen, Schädeltraumen, Intoxikationen und Infektionen, aber auch durch eine besondere endogene Veranlagung im Sinne einer gesteigerten Labilität des biochemischen Gleichgewichtes bedingt sein. So könnte man sich vorstellen, daß dieselben Schädlichkeiten, welche durch die infolge des Lebensprozesses entstehenden giftigen Stoffwechselprodukte des Körpers geliefert werden, ein normal widerstandsfähiges Gehirn nicht weiter alterieren, dagegen bei einem endogen minderwertigen Assoziationsorgan (z. B. infolge Labilität des Biochemismus) bereits Störungen hervorrufen, die infolge der Andauer der auslösenden Ursache eventuell zu später immer tiefer greifenden Schädigungen des ganzen Hirnmechanismus führen, wobei dann nicht bloß jene besprochenen höchsten psychischen Funktionen Schaden leiden werden, sondern in einzelnen Fällen die Schädigung zu einer die Assoziationssysteme in Mitleidenschaft ziehenden und daher zu unheilbaren psychischen Defektsymptomen (Demenz) führenden Krankheitsursache wird (Autointoxikationstheorie der Dementia praecox; Berze, Uber die hereditären Beziehungen der Dementia praecox). Stärker wirkende und akuter einsetzende exogene Schädlichkeiten werden natürlich auch imstande sein, den Biochemismus eines a priori rüstigen Gehirnes schwer zu alterieren und so die auslösende Ursache für akute Psychosen werden, bei denen wir dann meist auch das Bild schwerster Assoziationsstörungen, einer Lockerung und Auflösung des normalen Assoziationsgefüges, vor uns haben mit den mannigfaltigsten Reiz- und Lähmungserscheinungen seitens des Assoziationsorganes (akute Verwirrtheitszustände und Delirien bei Intoxikationen, Infektionen und Erschöpfungszuständen). 
Dabei werden wir bei genauerem Studium des Verlaufes der einzelnen Psychosen konstatieren können, daß im Beginne der schweren akuten Verwirrtheitszustände, also jener Gruppe von Geistesstörungen, deren Krankheitsursache nach dem Vorhergehenden als eine intensiv wirkende und auch ein früher ganz gesundes Gehirn schwer alterierende angenommen werden muß, vorzugsweise jene Symptome im Vordergrunde des klinischen Bildes stehen, die wir sonst in anderen Fällen, wo weniger intensiv wirkende exogene Schädlichkeiten infolge minderwertiger Hirnanlage bereits zu geistigen Störungen führen, als Krankheitserscheinungen einer mehr chronisch verlaufenden Psychose kennen. So ist es ja allbekannt, daß sehr häufig die akuten Verwirrtheitszustände sich mit einer paranoiden oder depressiven Phase einleiten, worauf erst die schwerere Störung der vollkommenen Dissoziation und Dissolution des Assoziationsgefüges, also klinisch das Bild der Verwirrtheit einsetzt. Gemäß unseren obigen Auseinandersetzungen, wonach die primäre und wesentliche Rolle im Psychismus dem Willen - sei es als Apperzeption, sei es als Endergebnis unserer Stimmungslage und Affekte und damit unseres Gefühls- und Trieblebens - zufällt und der eigentliche Intellekt jmmer nur eine sekundäre Rolle spielt, werden wir auch erwarten müssen, daß bei den Geistesstörungen ganz allgemein der Wille viel häufiger und schon bei leichteren Formen derselben in Mitleidenschaft gezogen und so dessen Alteration zur eigentlichen psychologischen Grundlage der Psychose wird, während die rein intellektuellen Funktionen viel seltener Schaden leiden, wenn aber, dann auch bereits viel schwerere, tiefer greifendere psychische Störungen (Verwirrtheit, Demenz) vorliegen, weil eben in diesen Fällen neben oder abgesehen von dem Baumeister (Wille, Apperzeption) auch bereits das Baumaterial (Vorstellungen, Begriffe, Assoziationsreiben, Gedächtnis) schwer geschädigt und eventuell ganz unbrauchbar geworden ist. Diese unmittelbar aus unserer Auffassung über den Ablauf der psychischen Funktionen sich ergebende Erwartung finden wir auch in der Klinik der Psychosen bestätigt. Wenn wir von den den Psychosen zugrunde liegenden psychologischen Grundphänomenen ausgehen wollen, so werden wir eine große Anzahl von Geistesstörungen finden, in denen sich die ganzen komplizierten Erscheinungen der Psychose ungezwungen auf ein psychopathologisches Grundsymptom zurückführen lassen, wobei wir wieder konstatieren werden können, daß in der großen Mehrzahl der Fälle dieses einer Willensstörung im weiteren Sinne gleichkommt, ja wir werden finden, daß die Willensstörung eben das ganze Wesen der Psychose ausmacht, während Störungen des Intellekts (Gedächtnis, Vorstellungs-, Begriffsschatz) entweder ganz fehlen oder nur eine sekundäre Rolle spielen.

Wir wollen nun versuchen, ausgehend von der theoretischen Trennung der Bewußtseinstätigkeit in Funktionen des Willens und des Intellekts, 
die uns aus der Klinik der Psychosen im allgemeinen bekannten Krankheitserscheinungen auf psychopathologische Grundstörungen zurückzuführen. Die Willensfunktionen in unserem Sinne umfassen nach dem Gesagten die innere und äußere, aktive und passive Apperzeption (Wundt) und die Affekte in einer bestimmten Entwicklungsphase, in der sie bereits den motorischen Impuls zum Handeln in sich bergen. Wir müßten demnach unter den Willensstörungen vorzugsweise die Apperzeptionsstörungen und die Affektstörungen im obigen Sinne unterscheiden. Berze hat bereits in seinen grundlegenden Arbeiten über die Primärsymptome bei Paranoia und Dementia praecox ausgeführt, daß es sich in beiden Fällen im wesentlichen um Störungen der passiven resp. aktiven Apperzeption und des Biotonus des Bewußtseins handelt. In paranoiden Krankheitsfällen wird infolge Störungen der passiven Apperzeption in dem Kranken das Gefühl des Erleidens durch die Sinneseindrücke und damit die psychologische Grundlage zu den weiteren paranoiden Wahnideen geschaffen, in vielen Fällen von Dementia praecox kommt es zu einer allgemeinen Hypotonie des Bewußtseins und sekundärem Nachlassen der aktiven apperzeptiven Tätigkeit, woraus Berze wieder die Bewußtseinsdissoziation (alternierendes Bewußtsein, Hervortreten der Nebenreihen, die als impulsive, ,fremde“ Einfälle und Handlungen beim Kranken auftauchen usw.) ableitet.

Diese Hypotonie des Bewußtseins ist natürlich, wie Berze ebenfalls erwähnt, kein der Dementia praecox charakteristisches Krankheitssymptom, sondern wird bei allen möglichen klinischen Krankheitsbildern zutage treten, sofern nur Bedingungen gegeben sind, die zu einem Absinken des Biotonus des Bewußtseins Anlaß geben. Dieses Nachlassen des Bewußtseinstonus (Absinken des Kraftindex des Bewußtseinsfeldes) empfinden die Kranken einerseits als Erschwerung im Denken, das bis zum vollkommenen Stupor führen kann, anderseits infolge Auftauchens der oben erwähnten psychischen Nebenreihen aus dem Unterbewußtsein in das Hauptbewußtsein, das eben bei der Abnahme seines Tonus nicht mehr imstande ist, derartige psychische Gebilde ,aus der Tiefe“" zurückzudämmen, als eine Beeinflussung ihres eigenen ihnen bewußten Willens durch fremde Macht. Es stellt sich dann als notwendige Folge bei derartigen Kranken das Gefühl ein, daß bestimmte Handlungen, die sie ausführen „müssen“, gar nicht ihrem eigenen selbstbewußten Ich entsprechen, sondern auf "Suggestion“, fremder Beeinflussung beruhen, weil eben, wie erwähnt, bei diesen im Unterbewußtsein, in den ,Nebenreihen" zur Reife gelangten psychomotorischen Akten nur die Endglieder als fertige psychische Gebilde ins Hauptbewußtsein treten und da vom Ichbewußtsein (Hauptbewußtsein) als fremde Gedanken und Impulse zu Handlungen empfunden werden. Mit dem Absinken des Biotonus des Bewußtseins und der aktiven Apperzeption ist recht häufig 
auch eine Störung im Ablauf des passiven Apperzeptionsvorganges verbunden, wodurch psychologisch nach den Ausführungen Berzes die paranoiden Symptome (paranoide Wahnideen, paranoide Ratlosigkeit) entstehen müssen. Wenn wir nur die vielfachen psychopathologischen Erscheinungen ins Auge fassen, die sich ungezwungen aus den vorerwähnten Apperzeptionsstörungen im Verein mit einer Hypotonie des Bewußtseins ergeben, können wir uns theoretisch nahezu alle Varianten im klinischen Verlaufe der subakut und chronisch verlaufenden Psychosen, die heute der Dementia praecox zugezählt werden, konstruieren. Denkerschwernis bis zur Hemmung und Stupor, paranoide Ideen, paranoide Ratlosigkeit, Interesselosigkeit, Autismus, mangelnde Hemmung der Affekte durch Erlahmen der apperzeptiven Tätigkeit, Auftauchen von Zwangsimpulsen, ,,plötzlichen“, unmotivierten Einfällen, Querhandlungen, ,raptusartige" motorische Akte, wären beiläufig diejenigen psychopathischen Erscheinungen, die sich aus den Apperzeptionsstörungen und der Hypotonie des Bewußtseins ableiten lassen und die gewöhnlich der betreffenden Psychose das charakteristische Gepräge geben.

Berücksichtigen wir noch die besondere Art der Affektstörungen, die derartigen Kranken zu eigen ist, woraus sich wieder die Regellosigkeit, Unverständlichkeit, die Inkoordination zwischen Affekt und Handeln erklären läßt, so wird immer mehr Klarheit und psychologisches Verständnis in das bunte Gewirr psychopathologischer Krankheitserscheinungen, wie sie uns in dem klinischen Bilde derartiger Psychosen entgegentreten, hineingebracht.

Die erwähnten Störungen der psychischen Höchstfunktionen des Assoziationsorganes können natürlich bei den verschiedensten klinischen Krankheitsbildern in Erscheinung treten und werden einmal bedingt sein dadurch, daß ein schon in der Anlage etwas minderwertiges Assoziationsorgan, wie oben erwähnt, bereits den Giften des normalen, zum Lebensprozeß notwendigen Stoffwechselumsatzes und eventueller Dysfunktion der endokrinen Drüsen erliegt und so funktionell erlahmt (Dementia praecox); in anderen Fällen werden die psychischen Krankheitserscheinungen dadurch zustande kommen, daß das lebende Hirngewebe infolge beginnender allgemeiner cerebraler Zirkulationsstörungen in seinem Biotonus nachläßt (paranoide Fälle im Praesenium), wobei die daraus entstehenden funktionellen Störungen unter entsprechender schonender und roborierender Therapie sich wieder ausgleichen oder aber, wenn die anatomischen Grundlagen (Atheromatose) für das $\mathrm{Zu}$ standekommen derartiger psychischer Symptome zu vorgeschritten oder gar progressiv sind, nur als Vorläufer schwerer nekrobiotischer, auch die Assoziationsreihen und die materiellen Residuen für das Vorstellungsleben schädigender Veränderungen im Sinne einer Dementia senilis be- 
wertet werden müssen. Auch die chronisch so deletär wirkende Noxe, die der paralytischen Stoffwechselerkrankung zugrunde liegt, kann im Beginn des Leidens die erwähnten Störungen hervorrufen, ebenso bestimmte cbronische Intoxikationen (Alkoholismus). Interessant sind dies bezüglich jene Fälle von progressiver Paralyse, wo sich das schwere Hirnleiden zuerst durch das Auftreten eines transitorischen psychischen Dämmerungszustandes kundgibt.

So sehen wir also, daß auf der einen Seite Affekt, Wille und Psychomotilität, zusammengehören, wesensgleiche und einheitlich funktionierende Faktoren im Psychismus, zugleich das wirklich aktive Prinzip unseres ganzen Geisteslebens darstellen, während andererseits der Intellekt solange ein Caput mortuum bleibt, als er nicht durch die Apperzeption (Wille) angeregt und in Funktion versetzt wird. Wenn der Mechanismus der Affekte und der zum Teil dadurch bewirkten Apperzep! ionserregung gestört ist, wird sich bei dem Subjekt als erster Grad affektiver Störung eine Interesselosigkeit kundgeben, die als Leere ,im Kopfe", als Willens- und Denkunfähigkeit empfunden wird. Bei schwereren derartigen Störungen in den somatischen Grundlagen der Affekte und mangelnder assoziativer Einwirkung derselben auf das Vorstellungsleben werden wir die bekannten psychopathologischen Symptome psychomotorischer Hemmung, die bis zum Stupor sich steigern kann, erkennen. Beim Infunktiontreten des rein somatischen Apparates der Affekte können wir bei derartigen Kranken wohl noch scheinbar Affektäußerungen wahrnehmen, aber ohne eigentliches psychisches Korrelat, ohne psychischen Inhalt, rein äußerlich unter dem Bilde des Affektvorganges ablaufend, weil eben die Affektempfindung nicht mehr imstande ist, reizauslösend auf das Vorstellungsleben assoziativ einzuwirken.

Als zweite große Hauptgruppe psychopathologischer Krankheitssymptome wären dann die intellektuellen Störungen sensu strictori zu erwähnen. Störungen in den perzeptiven Funktionen unseres psychischen Organs führen notwendigerweise zu schweren formalen intellektuellen Krankheitserscheinungen, da ja unser ganzer Intellekt nur auf der richtigen Perzeption der Außenwelt basiert. Krankhafte Erscheinungen beim Ablauf des Wahrnehmungsvorganges (Halluzinationen), Schädigungen des materiellen Substrates des Gedächtnisses, Lähmung und Destruktion in den Assoziationsbahnen und Ganglienzellen, Lockerung der Assoziationsgefüge führen natürlich insgesamt zu schweren intellektuellen Störungen, zu formalen Denkstörungen, Bewußtseinsverfälschungen oder Demenzerscheinungen.

Wir hätten damit eine Art Einteilungsgrund für die gesamten Psychosen gefunden, auf Grund dessen das ganze Gebiet der Psychiatrie in Intellekt- und Affekt- bzw. Willenspsychosen zerfallen würde. Wie 
bekannt, hat Ziehen bereits diese Einteilung der Geistesstörungen in Intellekt- und Affektpsychosen anerkannt, nur mit dem Zusatze, daß er Willenspsychosen im engeren Sinne eben gemäß seiner psychologischen Grundauffassungen gar nicht anerkennen will. Wir haben aber gerade in der Affektivität einen Entstehungsmechanismus für unsere psychische Aktivität kennen gelernt und den Willen gewissermaßen nur als eine Entwicklungsphase der ersteren betrachtet, sofern eben unsere Gefühle und Affekte zum treibenden, zur Aktivität drängenden Momente in unserem Bewußtseinsorgane geworden sind, in welchem Falle wir eben nicht mehr von Affekten, sondern von Willensregungen sprechen. Wenn wir daher den Ausdruck Affektpsychosen beibehalten, so wollen wir darunter nicht nur die reinen affektiven Störungen unseres Seelenlebens, sondern ebenso auch die Störungen der Willensfunktionen im engeren Sinne des Wortes verstanden wissen, auf welch letztere wir gerade beim krankhaften Handeln der Geistesgestörten den größten Wert legen möchten. Natürlich werden wir bei einer speziellen Psychose immer Störungen der einen Sphäre mit solchen der anderen verquickt finden, es werden also in jedem Falle von Geistesstörung meist Störungen des Affektlebens und der Willensfunktionen ebenso wie rein intellektuelle (formale) Störungen aufzudecken sein, immer würden wir aber ein ausschlaggebendes Utberwiegen entweder der Affekt-Willensstörungen oder der rein intellektuellen Ausfallssymptome konstatieren können. Durch die Anerkennung gerade dieser großen Gruppe der Affektpsychosen in obigem Sinne könnte sich unsere Wissenschaft auch viel praktischen Gewinn versprechen; denn wenn die Lehre von reinen Willensstörungen in unserem Sinne auch bei den in psychiatrischen Fragen in Betracht kommenden forensisch-juridischen Faktoren sich das Anerkennungsrecht erwerben könnte, dann würden auch diese letzteren erkennen, daß ein psychopathologisches Gebaren trotz intaktester Intelligenz und bei vollkommener Klarheit und Orientierungsfähigkeit des Patienten vorliegen kann, und es würde dann selbstverständlich von juridischer Seite nicht mehr soviel Gewicht auf das Vorhandensein rein intellektueller Krankheitssymptome oder formaler Bewußtseinsstörungen (Bewußtlosigkeit, Gedächtnisschwäche, Demenz, Wahnideen) gelegt werden und so ein bis jetzt noch immer heikles Gebiet unangenehmer Kontroversen zwischen Psychiatern und Juristen nahezu ganz aus der Welt geschafft sein. Gerade bei der Dementia praecox haben wir ja in vielen Fällen gar keine eigentliche Demenz vor uns, nämlich eine Demenz im Sinne von grob intellektuellen, den Bewußtseinsinhalt betreffenden Ausfallssymptomen, sondern vielmehr eine vorgetäuschte Pseudodemenz, die eben ihren letzten Grund in Willensstörungen hat, in dem Fehlen des einheitlichen regulativen Prinzips des ganzen Psychismus, in einer Störung der Bewußtseinstätigkeit (Bewußtseinsspaltung, Auftreten von 
Nebenreihen usw.) infolge Absinkens des Biotonus des Bewußtseinsfeldes und sekundärem Erlahmen der Apperzeption (Berze).

Zum Schlusse möchte ich mir noch die Bemerkung erlauben, daß es ebenfalls im Interesse der Entwicklung unserer Wissenschaft gelegén wäre, wenn sich deren Vertreter nicht so sehr in detaillierter Kleinarbeit erschöpfen würden, wobei man notgedrungen immer mehr den weiteren Blick für das gesamte Ganze verlieren muß, sondern ab und zu auch wieder einmal das Werk irgendeines wirklichen großen Denkers zur Hand nehmen und zu ihrer Erholung es durchblättern würden. Ich bin überzeugt, daß nicht wenige daraus auch für psychologisch-psychiatrische Fragen Anregung und die Lösung mancher Zweifel finden würden. Eingedenk dieser hier gebrachten Aufmunterung zum Studium psychologisch-philosophischer Schriften, wobei man nie den selbständigen Standpunkt des naturwissenschaftlich denkenden Mediziners aufzugeben braucht, soferne man nur Anregung von der Fülle der in solchen Werken oft niedergelegten Gedanken zu weiterem selbständigen Denken sucht und sich nicht dogmatisch an irgendein philosophisches System anklammern will, glaube ich meine Ausführungen nicht besser beschließen zu können, als indem ich, gerade zum Beweise meiner letzten Behauptung, einen aus der Reihe dieser Weltfremden, Suchenden das Wort selbst führen lasse, um damit zu zeigen, in wie scharfen und klaren Ausdrücken ein nicht zur Fachgilde Gehöriger, aber an tiefes, ernstes Denken gewöhnter Kopf auch in psychologischen Fragen die Sprache zu meistern versteht.

So legt Schopenhauer, der Vertreter der Lehre vom Primat des Willens (im metaphysischen Sinne), in dem Kapitel über Gedankenassoziationen folgende Ansicht darüber nieder: ,Uberhaupt ist in der Wirklichkeit der Gedankenprozeß unseres Innern nicht so einfach wie die Theorie desselben, da hier vielerlei ineinandergreift. Vergleichen wir, um uns die Sache zu veranschaulichen, unser Bewußtsein mit einem Wasser von einiger Tiefe, so sind die deutlich bewußten Gedanken bloß die Oberfläche, die Masse hingegen ist das Undeutliche, die Gefühle, die Nachempfindungen der Anschauungen und des Erfahrenen überhaupt, versetzt mit der eigenen Stimmung unseres Willens, welcher der Kern unseres Wesens ist. Die Masse des ganzen Bewußtseins ist nun mehr oder weniger nach Maßgabe der intellektuellen Lebendigkeit in steter Bewegung, und was infolge dieser auf die Oberfläche steigt, sind die klaren Bilder der Phantasie oder die deutlichen, bewußten, in Worten ausgedrückten Gedanken und die Beschlüsse des Willens. Selten liegt der ganze Prozeß unseres Denkens und Beschließens auf der Oberfläche, d. h. besteht in einer Verkettung deutlich gedachter Urteile, obwohl wir dies anstreben, um uns und anderen Rechenschaft geben zu können; gewöhnlich aber geschieht in der dunklen Tiefe die Rumination des von 
außen erhaltenen Stoffes, durch welche er zu Gedanken umgearbeitet wird, und sie geht beinahe so unbewußt vor sich, wie die Umwandlung der Nahrung in die Säfte und Substanz des Leibes. Daher kommt es, da $\$$ wir oft vom Entstehen unserer tiefsten Gedanken keine Rechenschaft geben können; sie sind die Ausgeburt unseres geheimnisvollen Innern. Urteile, Einfälle, Beschlüsse steigen unerwartet und zu unserer eigenen Verwunderung aus jener Tiefe auf.

Das Bewußtsein ist die bloße Oberfläche unseres Geistes, von welchem, wie vom Erdkörper, wir nicht das Innere, sondern nur die Schale kennen. Was aber die Gedankenassoziation selbst in Tätigkeit versetzt, ist in letzter Instanz oder im Geheimen unseres Innern, der Wille, welcher seinen Diener, den Intellekt, antreibt, nach Maßgabe seiner Kräfte Gedanken an Gedanken zu reihen, das Ähnliche, das Gleichzeitige zurückzurufen, Gründe und Folge zu erkennen; denn im Interesse des Willens liegt es, daß überhaupt gedacht werde, damit man möglichst orientiert sei für alle vorkommenden Fälle usw." -

Das eben Rezitierte soll nur eine kleine Stichprobe für meine oben aufgestellte Behauptung liefern; in ebenso interessanter und klarer Weise finden wir die verschiedensten psychologischen Fragen behandelt in anderen Kapiteln seines Hauptwerkes (z. B. von den wesentlichen Unvollkommenheiten des Intellekts, vom Verhältnis der anschauenden zur abstrakten Erkenntnis, vom Primat des Willens, im Selbstbewußtsein, objektive Ansicht des Intellekts usw.). 